\title{
Screening and Verification of Differentially Expressed Long Non-coding RNAs in the Peripheral Blood of Patients With asthma
}

\section{OPEN ACCESS}

Edited by: Roberto Paganelli, Institute for Advanced Biologic Therapies, Italy

Reviewed by: Asunción García-Sánchez, University of Salamanca, Spain Damiano D'Ardes, SS Annunziata Polyclinic Hospital, Italy

*Correspondence: Jingcheng Dong jcdong2004@126.com Ying Wei

weiying_acup@126.com

${ }^{\dagger}$ These authors have contributed equally to this work

Specialty section: This article was submitted to Respiratory Pharmacology, a section of the journal

Frontiers in Pharmacology

Received: 12 December 2021 Accepted: 19 January 2022

Published: 22 February 2022

Citation:

Ma C, Wang S, Cao Y, Tang W, Wuniqiemu $T$, Teng F, Zhu $X$, Wei $Y$ and Dong J (2022) Screening and Verification of Differentially Expressed

Long Non-coding RNAs in the

Peripheral Blood of Patients

With asthma.

Front. Pharmacol. 13:834009. doi: 10.3389/fphar.2022.834009

\author{
Cheng Ma ${ }^{1,2 \dagger}$, Shiyuan Wang ${ }^{1,2 \dagger}$, Yuxue Cao ${ }^{1,2}$, Weifeng Tang ${ }^{1,2}$, Tulake Wuniqiemu ${ }^{1,2}$, \\ Fangzhou Teng ${ }^{1,2}$, Xueyi Zhu ${ }^{1,2}$, Ying Wei ${ }^{1,2 *}$ and Jingcheng Dong ${ }^{1,2 *}$
}

${ }^{1}$ Department of Integrative Medicine, Huashan Hospital, Fudan University, Shanghai, China, ${ }^{2}$ Institutes of Integrative Medicine, Fudan University, Shanghai, China

Growing evidence suggests that long non-coding RNAs (IncRNAs) play a key role in the pathogenesis of asthma. Although some differentially expressed IncRNAs have been identified in asthmatic patients, many asthma-related IncRNAs have not been annotated. In the present study, six patients and three healthy subjects were randomly selected from 34 asthmatic patients and 17 healthy subjects. Second-generation high-throughput sequencing was performed on their peripheral blood samples. There were 1,137 differentially expressed IncRNAs in the asthma patients compared to in the healthy controls, of which 485 were upregulated and 652 were downregulated. The top 30 enriched GO and KEGG terms were identified, and the cytosolic ribosome (GO:0022626) and ribosome (hsa03010) were associated with the most differentially expressed IncRNAs. The top 10 differentially expressed IncRNAs associated with asthma were verified by an IncRNA-mRNA co-expression network and RT-qPCR. Seven of the these (NONHSAT015495.2, MSTRG.71212.2, NONHSAT163272.1, NONHSAT181891.1, NONHSAT190964.1, ENST00000564809, and NONHSAT076890.2) were downregulated in the peripheral blood of asthmatic patients, which was consistent with the sequencing results. Three patients and three healthy subjects were randomly selected from the remaining subjects to verify these seven IncRNAs by RT-qPCR, which further confirmed the sequencing results. Public database GSE106230 was also in agreement with the FPKM (Fragments Per kilobase of exon model per Million mapped reads) trends of ENST00000564809, NONHSAT015495.2, NONHSAT181891.1, and NONHSAT190964.1. In conclusion, the present study identified seven IncRNAs that may serve as potential biological markers for asthma.

Keywords: asthma, full transcriptome sequencing, bioinformatics, IncRNAs, mRNAs

\section{INTRODUCTION}

Asthma is a heterogeneous disease characterized by chronic airway inflammation, airway hyperresponsiveness, and airway remodeling (Hammad and Lambrecht, 2021), and affects more than 300 million patients worldwide (Côté et al., 2020). The pathogenesis of asthma remains unclear. It is generally thought that asthma is caused by a combination of genetic and environmental factors, 
resulting in an imbalance in the ratio of T helper cells (Th)1/Th2 and Th17/Treg cytokines and other factors in the airway, resulting in persistent inflammation and hyperresponsiveness (Kudo et al., 2012; Fahy, 2015).

Long non-coding RNAs (lncRNAs) are a type of non-coding RNA that are $>200$ nucleotides in length, and can interact with proteins, DNA, or RNA (Chen and Shan, 2020; Vydzhak et al., 2020). LncRNAs are involved in the regulation of many biological processes, including chromatin modification, transcriptional activation and inhibition, post-transcriptional modification, and nuclear transport, and as a result play a role in many important biological processes, including cell differentiation and development (Lodde et al., 2020). Recent studies have shown that lncRNAs play a key role in the pathogenesis of asthma. For instance, the overexpression of IncRNA-MALAT1 is associated with asthma patients, and its expression was negatively correlated with the expression of Th1/Th2 (Liang and Tang, 2020). Another lncRNA, MEG3, is a competing endogenous RNA that regulates the balance of Treg/Th17 in asthma patients (Qiu et al., 2019).

With the recent development and continuous improvement of second-generation high-throughput sequencing technology, current transcriptomics research depends on accurate and rapid screening for relevant lncRNAs that are related to specific diseases. An increasing number of lncRNAs have been functionally annotated. Although studies have reported some differences in lncRNAs in asthma patients during both childhood (Zheng et al., 2020) and adulthood (Gál et al., 2020), there are many lncRNAs with an unclear relationship to asthma that require investigation and annotation. In the present study, we aimed to perform whole transcriptome sequencing in patients with asthma using second-generation high-throughput sequencing to identify dysregulated lncRNAs that are associated with asthma. We will then perform GO and KEGG enrichment analysis to identify potential biological functions associated with the dysregulated lncRNAs. In doing so, we hope to identify an lncRNA signature for asthma that may have clinical diagnostic or prognostic applications, and may alleviate the burden on healthcare systems by enabling timeous and effective interventions.

\section{METHODS}

\section{Subjects \\ Study Cohort}

Subjects were patients with asthma who attended the Outpatient of Integrative Medicine, Huashan Hospital, Fudan University (from 1 November 2019 to 31 January 2020), and healthy volunteers who were recruited through clinical research recruitment advertisements. According to the inclusion and exclusion criteria (detailed below), a total of 34 subjects in the asthma group and 17 subjects in the healthy control group were included. Each subject signed an informed consent form.

\section{Diagnostic Criteria for Asthma}

The diagnostic criteria for asthma were based on the 2016 Chinese Medical Association Respiratory Diseases Association Bronchial Asthma Prevention and Control Guidelines. 1) Clinical symptoms and signs of typical asthma: recurrent wheezing, shortness of breath, with or without chest tightness or coughing, especially at night and in the morning, and often attacks after exposure to allergens such as cold air, physical or chemical irritation, upper respiratory tract infection, or exercise. Sporadic or diffuse wheezing can be heard in both lungs during attack, with a predominant expiratory phase and prolonged expiratory phase. The above symptoms or signs are relieved spontaneously or after treatment. 2) Objective inspection of variable airflow limitation: 1) a positive bronchial diastolic test with an increase in FEV between 1\%>12\% after bronchodilator inhalation, and an increase in absolute FEV between $1>200 \mathrm{ml}$ 2) A positive bronchial provocation test. 3) Peak expiratory flow (PEF) average daily diurnal variation rate of $>10 \%$ or weekly PEF variation rate of $>20 \%$. 4) The patient meets the "typical clinical symptoms and signs of asthma," and has any of the "objective examinations of variable airflow limitation," and wheezing, shortness of breath, chest tightness, or cough caused by other diseases are excluded.

\section{Inclusion and Exclusion Criteria}

\section{Selection Criteria for Asthma Patients}

(1) Diagnosed as bronchial asthma according to 2016 Respiratory Diseases Association Bronchial Asthma Prevention and Control of Chinese Medical Association and Guidelines and 2019 Global Initiative for Asthma (GINA) guidelines (2) The selected age range was 30-55 years, regardless of gender. (3) No history of respiratory diseases, except for asthma. (4) No upper/ lower respiratory tract infections and no antibiotic treatment within 4 weeks; no oral/intravenous glucocorticoid treatment or other respiratory medication except Inhaled Corticosteroids/Long-Acting Beta2 Agonist (ICS/LABA) and Short-Acting Beta2-Agonists (SABA) within 4 weeks. (5) No smoking history or had quit smoking for more than 12 months. (6) Voluntarily participated in the study and signed informed consent forms.

\section{Selection Criteria for Healthy Subjects}

(1) The selected age range was 30-55 years, regardless of gender. (2) The physical examination results were normal, without any abnormal symptoms or signs. (3) Laboratory/imaging examination results were normal or abnormal, but had no clinically meaningful results. (4) No smoking history or had quit smoking for more than 12 months. (5) Voluntarily participated in the study and signed informed consent forms.

\section{Exclusion Criteria}

\section{Exclusion Criteria for Asthma Patients}

(1) Patients with severe persistent and acute exacerbation of asthma requiring oral or intravenous corticosteroid treatment. (2) Patients with COPD, bronchiectasis, interstitial lung disease, active tuberculosis, pulmonary embolism, pleural effusion, or other lung diseases. (3) Patients with cardiovascular, cerebrovascular, digestive, endocrine, kidney, urinary, blood, and other systemic diseases or malignant tumors. (4) Patients who received antibiotic treatment within 4 weeks of the study. (5) 
Patients requiring treatment with glucocorticoids or immunosuppressive agents. (6) Patients who have been pregnant or breast fed within 12 months (8) Patients participating in other clinical studies during the same period. (9) Patients who refused to sign informed consent forms.

\section{Exclusion Criteria for Healthy Subjects}

(1) Subjects who received antibiotics or glucocorticoid therapy within 4 weeks of the study. (2) Subjects who had been pregnant or had breast fed within 12 months. (3) Subjects participating in other clinical studies during the same period. (4) Subjects who refused to sign informed consent forms.

\section{Ethical Review}

This single-center, prospective, and exploratory clinical study was approved by the Ethical Review Committee of Huashan Hospital Affiliated to Fudan University. Ethical approval number (2019). Temporary Approval No (593). The study was registered with the China Clinical Trial Registration Center, clinical research registration number: ChiCTR2000031267. The study was performed according to the guidelines established by the Declaration of Helsinki.

\section{Research Methods Clinical Characteristics}

Detailed clinical consultations were conducted on asthma patients and healthy subjects, with a comprehensive physical examination performed, including body temperature, pulse, respiration, blood pressure, weight, and height. Medical histories, including allergies and other medical conditions, were obtained to confirm the asthma diagnosis. Routine blood, urine, liver and kidney function, blood sugar, peripheral blood EOS count, blood total IgE, lung function, and FeNO tests were performed for all subjects.

\section{Determination of Lung Function and FeNO}

Both lung function and FeNO tests were performed between 8: 00-11:00 a.m. Subjects were required to stop vigorous activities for $2 \mathrm{~h}$ before the test and stay at rest for $15 \mathrm{~min}$. Patients with asthma were tested before the administration of asthma control drugs. Both lung function and FeNO measurement were tested using the Pulmonary Function Room at Huashan Hospital Affiliated to Fudan University.

\section{Detection of Inflammatory Cytokines}

Venous blood $(4 \mathrm{ml})$ was collected from fasting subjects before 8: 00 a.m., and centrifuged at $4,000 \mathrm{rpm}$ for $10 \mathrm{~min}$ at room temperature to separate red blood cells and serum. The serum was removed and stored in $2 \mathrm{ml}$ Eppendorf tubes at $-80^{\circ} \mathrm{C}$. An ELISA was used to detect serum inflammatory factors (IL-4, IL-5, IL-8, IL-9, IL-13, IL-17A, IL-25, IL-33, and IFN- $\gamma$ ) and eotaxin.

\section{Total RNA Extraction, Library Construction and Whole} Transcriptome Sequencing

Venous blood $(2.5 \mathrm{ml})$ was collected from fasting subjects before 8:00 a.m. Each sample was collected in a disposable vacuum blood
RNA collection tube, mixed well, and stored at $-80^{\circ} \mathrm{C}$. An RNEasy micro kit and RNase-Free DNAse kit were used for the extraction and purification of total RNA, according to the manufacturer's instructions. A NanoDrop ND-2000 spectrophotometer and Agilent Biochemical Analyzer 2,100 were used to determine RNA quality by measuring RNA integrity number (RIN). The RIN values ranged from to 0 to 10 , with higher scores indicating better RNA integrity. RNA samples that passed the quality assessment were used as the templates for first and second strand cDNA synthesis. A Qubit ${ }^{\circledR} 2.0$ Fluorometer (Invitrogen, Q32866) was used to detect DNA concentration. A 2100 Bioanalyzer (Agilent Technologies) was used to detect the band distribution, and then cluster generation and firstdirection sequencing primer hybridization were performed on the cBot for the Illumina sequencing platform, according to the manufacturer's instructions. The Illumina sequencing reagents were prepared according to manufacturer's instructions, and a patterned flow cell was used for improved cluster generation. Paired-end sequencing was performed using Illumina software, with real-time data analysis.

\section{Bioinformatics Analysis}

Seqtk was used to filter the original data to obtain clean reads for data analysis. The spliced mapping algorithm HISAT2 (version 2.0.4) was used for genome mapping of preprocessed sequences. The human GRCh37 assembly (Ensembl) was used for genome mapping. StringTie software (version 1.3.0) was used to count the number of fragments corresponding to each gene after HISAT2 comparison, and then normalized to the trimmed mean $M$ values (TMM) (fragments per kilobase of exon model per million mapped reads, FKPM) for each gene.

The edgeR $\mathrm{R}$ package was used to comparatively analyze the differentially expressed genes between the sample groups. After obtaining the $p$-value, multiple testing correction was applied, and the $p$-value threshold was determined by controlling the false discovery rate (FDR), and expressed as a q-value. The fold change (FC) was calculated based on the FPKM value. Cutoffs of q-value $\leq 0.05$, and FC $\geq 2$ were applied to filter for differentially expressed genes. The differentially expressed genes (DEGs) were mapped to the GO database, and the number of genes corresponding to each entry was calculated. KEGG pathway enrichment analysis was performed similarly for the DEGs. Differentially expressed lncRNAs were sorted in descending $\log 2 \mathrm{FC} \mid$ value, and the top 50 dysregulated IncRNAs were identified. KEGG pathways related to asthma with a large number of differentially expressed genes were selected, and the mRNAs enriched in these pathways were selected to generate an lncRNA-mRNA co-expression network and identify biologically relevant lncRNAs.

\section{Real-Time Quantitative PCR}

PCR primers were designed using Primer Express 3.0.1 software, synthesized by Sangon Biotech Co., Ltd (Shanghai, China) and verified by Primer-BLAST. The $2^{-\Delta \Delta C T}$ method was used to analyze the relative changes in gene expression, with GAPDH serving as the internal reference. Primer sequences and related information were included in Table $\mathbf{1}$. 
TABLE 1 | RT-qPCR Primer sequence.

\begin{tabular}{|c|c|c|}
\hline No. & Name & Sequence $\left(5^{\prime}\right.$ to $\left.3^{\prime}\right)$ \\
\hline \multirow[t]{2}{*}{1} & NONHSAT015495.2-F & GCACTCAGGAGGCATAGCAAA \\
\hline & NONHSAT015495.2-R & CGTGATCTCCCTGAGCTCATC \\
\hline \multirow[t]{2}{*}{2} & MSTRG.71212.2-F & GCCTCAGCTCACCATGTTGA \\
\hline & MSTRG.71212.2-R & AGGGCTGGCGATCTAGGAA \\
\hline \multirow[t]{2}{*}{3} & NONHSAT163272.1-F & CCAGTGATAGGCCTTGGTAGCT \\
\hline & NONHSAT163272.1-R & TCTACATGCATGCAGTCATTTCC \\
\hline \multirow[t]{2}{*}{4} & ENST00000564809-F & GCCAAAGCCAACAGCATCTT \\
\hline & ENST00000564809-R & GAATTGATAATGGCGTGAAACG \\
\hline \multirow[t]{2}{*}{5} & NONHSAT181891.1-F & TGACTGCAGTGATGAAGAAAGGA \\
\hline & NONHSAT181891.1-R & CTGGAGGTCCCTGCTGAATC \\
\hline \multirow[t]{2}{*}{6} & ENST00000615535-F & TGCCCGGAGCCTGAGA \\
\hline & ENST00000615535-R & TCCCCTGGCCCCATTT \\
\hline \multirow[t]{2}{*}{7} & NONHSAT190964.1-F & AGCATGCCACACATTGATGAA \\
\hline & NONHSAT190964.1-R & GTAATCCCGCCTTGCTITGA \\
\hline \multirow[t]{2}{*}{8} & NONHSAT076890.2-F & GCAGTAACCTTCATCTCTCTTCTATAGGT \\
\hline & NONHSAT076890.2-R & TTCAAAGCTGTCACTCTCCATGTT \\
\hline \multirow[t]{2}{*}{9} & GAPDH-F & CACCCTGTTGCTGTAGCCAAA \\
\hline & GAPDH-R & CACCCTGTTGCTGTAGCCAAA \\
\hline
\end{tabular}

TABLE 2 | Cohort clinical characteristics.

\begin{tabular}{llccc}
\hline & & Asthma group & Control group & $\boldsymbol{p}$ Value \\
\hline Gender & Male & $5(14.71 \%)$ & $11(16.71 \%)$ & $<0.001$ \\
& Female & $29(85.29 \%)$ & $6(35.29 \%)$ & \\
Age & $42.62 \pm 8.16$ & $40.76 \pm 6.01$ & 0.411 \\
$\mathrm{BMl}\left(\mathrm{kg} / \mathrm{m}^{2}\right)$ & $24.78 \pm 5.31$ & $23.79 \pm 3.29$ & 0.422 \\
\hline
\end{tabular}

\section{Statistical Analysis}

Statistical analysis was performed using SPSS 25.0 (IBM), and plots were generated using GraphPad 8.0 (GraphPad software, Inc). A $t$-test was used to analyze continuous variables that conformed to a normal distribution, and data are presented as the mean \pm standard deviation. Data that did not conform to a normal distribution were $\log 2$ transformed, and analyzed by $t$-test. If the $\log 2$ transformed data did not conform to a normal distribution, a Wilcoxon test was used, and the median and interquartile intervals were used for statistical analysis. A Chi-squared test was used to compare categorical variables. Differences were considered to be statistically significant at $p<0.05$.

\section{RESULTS}

\section{Cohort Clinical Characteristics}

The clinical characteristics of the asthma group $(n=34)$ and the healthy control group $(\mathrm{n}=17)$, such as age, sex, and BMI, were compared to exclude any potential sources of bias from the subsequent analysis. There was a difference in gender composition between the asthma group and the healthy control group $(p<0.001)$, with more female patients $(85.29 \%)$ than male patients $(14.71 \%)$ in the asthma group. There were no statistically significant differences between the two groups in terms of age and BMI $(p>0.05)$ (Table 2).
TABLE 3 | Comparison of lung function, FeNO and inflammatory cytokines.

\begin{tabular}{lccc}
\hline & Asthma group & Control group & $\boldsymbol{p}$ \\
\hline FEV1\% & $77.26 \pm 23.83$ & $94.85 \pm 10.44$ & $<0.001$ \\
FEV1/FVC\% & $82.68 \pm 15.88$ & $91.49 \pm 7.18$ & 0.009 \\
PEF\% & $76.27 \pm 27.44$ & $91.95 \pm 15.81$ & 0.013 \\
MEF25\% & $48.85(28.00-71.00)$ & $64.00(61.00-86.10)$ & 0.004 \\
MEF50\% & $59.96 \pm 32.83$ & $83.54 \pm 11.92$ & $<0.001$ \\
MEF75\% & $65.11 \pm 31.55$ & $89.24 \pm 13.13$ & $<0.001$ \\
MMEF75/25\% & $59.35 \pm 31.79$ & $80.64 \pm 14.40$ & 0.002 \\
FeNO & $25.00(19.00-43.00)$ & $19.00(15.00-22.00)$ & 0.009 \\
IgE & $215.96(45.43-446.61)$ & $51.72(40.46-119.27)$ & 0.010 \\
EOS & $170.00(130.00-320.00)$ & $110.00(88.00-190.00)$ & 0.032 \\
IL-4 & $0.02 \pm 0.01$ & $0.02 \pm 0.02$ & 0.284 \\
IL-5 & $0.49(0.34-0.79)$ & $0.63(0.49-0.79)$ & 0.329 \\
IL-8 & $14.71(9.59-27.13)$ & $6.43(5.07-10.40)$ & $<0.001$ \\
IL-9 & $8.59(3.09-14.75)$ & $10.14(6.32-17.24)$ & 0.342 \\
IL-13 & $1.31 \pm 0.88$ & $1.65 \pm 1.12$ & 0.245 \\
IL-17A & $3.31(0.96-17.67)$ & $2.04(0.71-14.50)$ & 0.453 \\
IL-25 & $3.13(2.17-5.26)$ & $2.17(1.29-3.13)$ & 0.1389 \\
IL-33 & $7.17(3.80-12.03)$ & $5.13(3.80-9.24)$ & 0.574 \\
IFN- $\gamma$ & $3.08(2.41-5.71)$ & $3.76(3.31-5.63)$ & 0.283 \\
& & &
\end{tabular}

\begin{tabular}{lccccc}
\hline \multicolumn{2}{l}{ TABLE 4 | Data processing results. } & \multicolumn{3}{l}{} \\
\hline No. & Raw reads & Clean reads & $\begin{array}{c}\text { Clean ratio } \\
\mathbf{( \% )}\end{array}$ & $\begin{array}{c}\text { rRNA ratio } \\
\mathbf{( \% )}\end{array}$ & $\begin{array}{c}\text { No. rRNA } \\
\text { pair }\end{array}$ \\
\hline 5 & & & & & \\
\hline 12 & $82,842,400$ & $74,321,932$ & 94.27 & 0.19 & $73,054,476$ \\
13 & $77,533,108$ & $73,731,201$ & 95.10 & 0.26 & $76,889,770$ \\
15 & $71,154,652$ & $67,801,944$ & 95.29 & 0.31 & $72,449,512$ \\
18 & $70,679,030$ & $66,843,822$ & 94.57 & 0.23 & $65,685,148$ \\
19 & $67,079,192$ & $61,883,695$ & 92.25 & 0.02 & $60,944,216$ \\
7 & $74,113,180$ & $70,950,110$ & 95.73 & 0.66 & $69,450,354$ \\
42 & $79,563,670$ & $75,110,559$ & 94.40 & 0.00 & $74,023,280$ \\
45 & $77,823,786$ & $73,605,030$ & 94.58 & 0.26 & $72,280,382$
\end{tabular}

Note: Clean ratio, (Clean reads/Raw reads) \%; rRNA, ratio, [(Clean reads - rRNA, trimed)/ Clean reads $] \%$.

\section{Comparison of Lung Function, FeNO and Inflammatory Cytokines}

We compared the lung function, FeNO, and inflammatory cytokine levels in the two groups, and found that the lung function indices (FEV1\%, FEV1/FVC\%, and PEF\%) and small airway function indexes (MEF25\%, MEF50\%, MEF75\%, and MMEF75/25\%) of the healthy subjects were significantly better than those in the asthma group $(p<0.05)$. Compared with healthy subjects, asthma patients had higher FeNO $(p<0.05)$, total blood $\operatorname{IgE}(p<0.05)$, peripheral blood EOS counts $(p<0.05)$, and serum IL-8 levels $(p<$ $0.01)$. The levels of inflammatory cytokines IL-17A, IL-25, IL-33, and eotaxin were higher in the asthma group than in the healthy control group, but these differences were not significant, and there were no significant differences in the levels of IL-4, IL-5, IL-9, and IL-13 between the two groups (Table 3 ).

\section{Total RNA, Sequencing Library Quality Inspection Results}

We randomly selected six patients with asthma and three healthy subjects from the two groups (the demographic information on 
TABLE 5 | Genome mapping results.

\begin{tabular}{|c|c|c|c|c|c|}
\hline No. & All reads & Mapped reads & $\begin{array}{c}\text { Mapped paired } \\
\text { reads }\end{array}$ & $\begin{array}{l}\text { Mapped unique } \\
\text { reads }\end{array}$ & $\begin{array}{c}\text { Mapping ratio } \\
(\%)\end{array}$ \\
\hline 5 & $74,023,280$ & $72,850,058$ & $72,290,900$ & $72,681,496$ & 98.42 \\
\hline 12 & $73,054,476$ & $71,617,326$ & $70,849,638$ & $71,362,228$ & 98.03 \\
\hline 13 & $76,889,770$ & $75,410,004$ & $74,587,740$ & $75,131,921$ & 98.08 \\
\hline 15 & $72,449,512$ & $71,065,744$ & $70,289,274$ & $70,815,998$ & 98.09 \\
\hline 18 & $66,603,148$ & $64,285,423$ & $63,711,042$ & $64,123,082$ & 96.52 \\
\hline 19 & $65,685,676$ & $62,310,147$ & $61,689,166$ & $62,133,069$ & 94.86 \\
\hline 7 & $72,280,382$ & $69,060,901$ & $68,400,236$ & $68,868,720$ & 95.55 \\
\hline 42 & $60,944,216$ & $59,382,248$ & $58,871,286$ & $59,213,248$ & 97.44 \\
\hline 45 & $69,450,354$ & $67,473,749$ & $66,879,836$ & $67,310,181$ & 97.15 \\
\hline
\end{tabular}

Note: Mapping ratio, Mapped reads/All reads; Mapped unique reads: Reads that match only one position in the reference Genome.

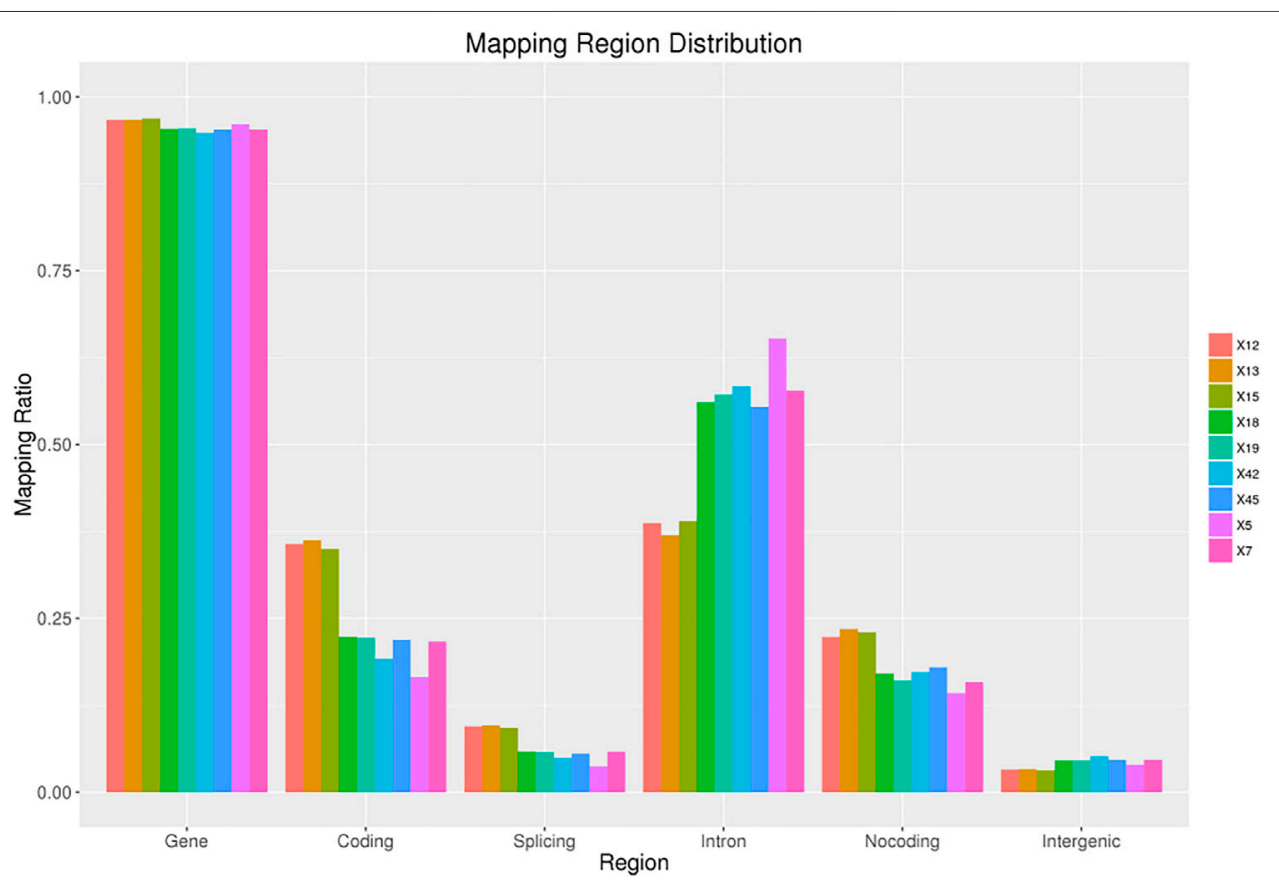

FIGURE 1|Regions distribution. The ratio of measured reads aligned to gene, coding, splicing, intron and non-coding region. Non-coding region includes statistics of total non-coding regions such as $5^{\prime}$ UTR, $3^{\prime}$ UTR, and non-coding RNA regions.

nine subjects were displayed in Supplementary Table S1A). The RIN values of all samples were $>7.0$ (Supplementary Table S1B), and the $28 \mathrm{~S} / 18 \mathrm{~S}$ ratios were all $>0.7$, indicating that the RNA was of good integrity. The Qubit ${ }^{\circledR} 2.0$ Fluorescence quantitative analysis results showed that the library was sufficiently high quality for sequencing (Supplementary Table S2). And the ratio of each base with a quality of more than 20 (Q20) was $\geq 85 \%$, and all samples met the quality control requirements (Supplementary Table S3).

\section{Data Processing and Genome Mapping}

The original sequences (raw reads) were filtered to obtain the effective sequences (clean reads) that could be used for data analysis (Table 4). We compared the sequences obtained with that of the reference sequence (Table 5 and Figure 1). The genome coverage distribution of each sample is shown in Figure 2, where the outermost circle is the genome, and each circle represents the chromosome coverage of a sample.

\section{Correlation Analysis}

We performed correlation co-efficient analysis and principal component analysis (PCA) using the FPKM expression data. According to the expression correlation heat map, samples 18 and 19 of the asthma group were highly similar to samples 7 and 45 of the healthy control group, and the correlation coefficients were all $>0.9$. Other samples in the asthma group $(5,12,13$, and 15) and the healthy control group $(7,42,45)$ had good intragroup similarity (Figure 3A). According to PCA, samples 7, 42, and 

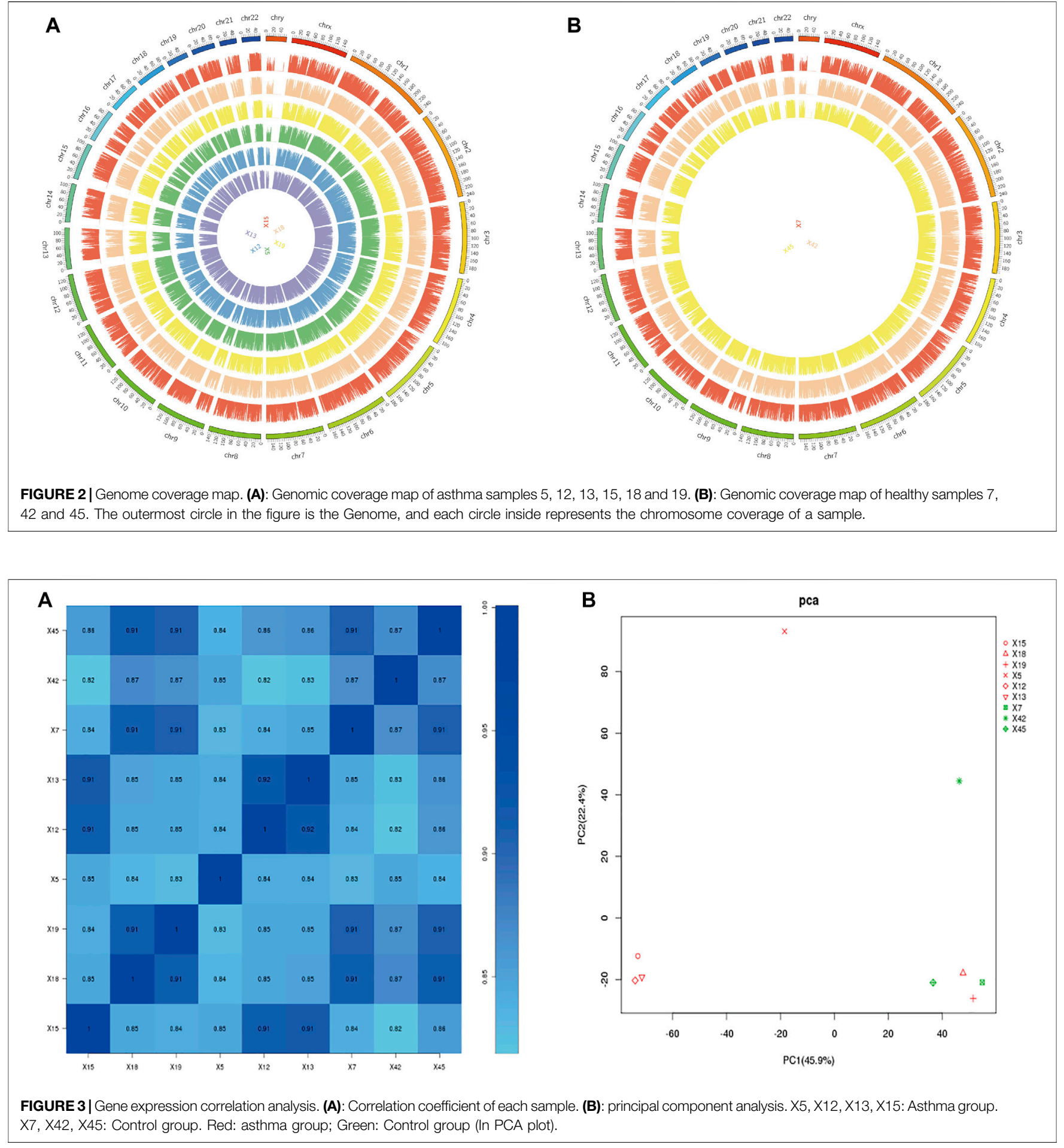

45 of the healthy control group had high similarity. Samples 5, 12, 13 , and 15 in the asthma group clustered together, while samples 18 and 19 were clear outliers (Figure 3B). To ensure the accuracy and reliability of the analysis results, samples 18 and 19 were therefore excluded, and four samples from the asthma group (5, 12,13 , and 15) and three samples from the healthy control group $(7,42,45)$ were selected for follow-up analysis.

\section{Analysis of Gene Expression in the Peripheral Blood}

2,290 mRNAs (994 up-regulated and 1,296 down-regulated) and 1,137 lncRNAs (485 up-regulated and 652 down-regulated) were differentially expressed between asthma group and healthy control group. The cluster analysis was used for the similarly expressed mRNAs and lncRNAs, and the heatmaps were drawn. 

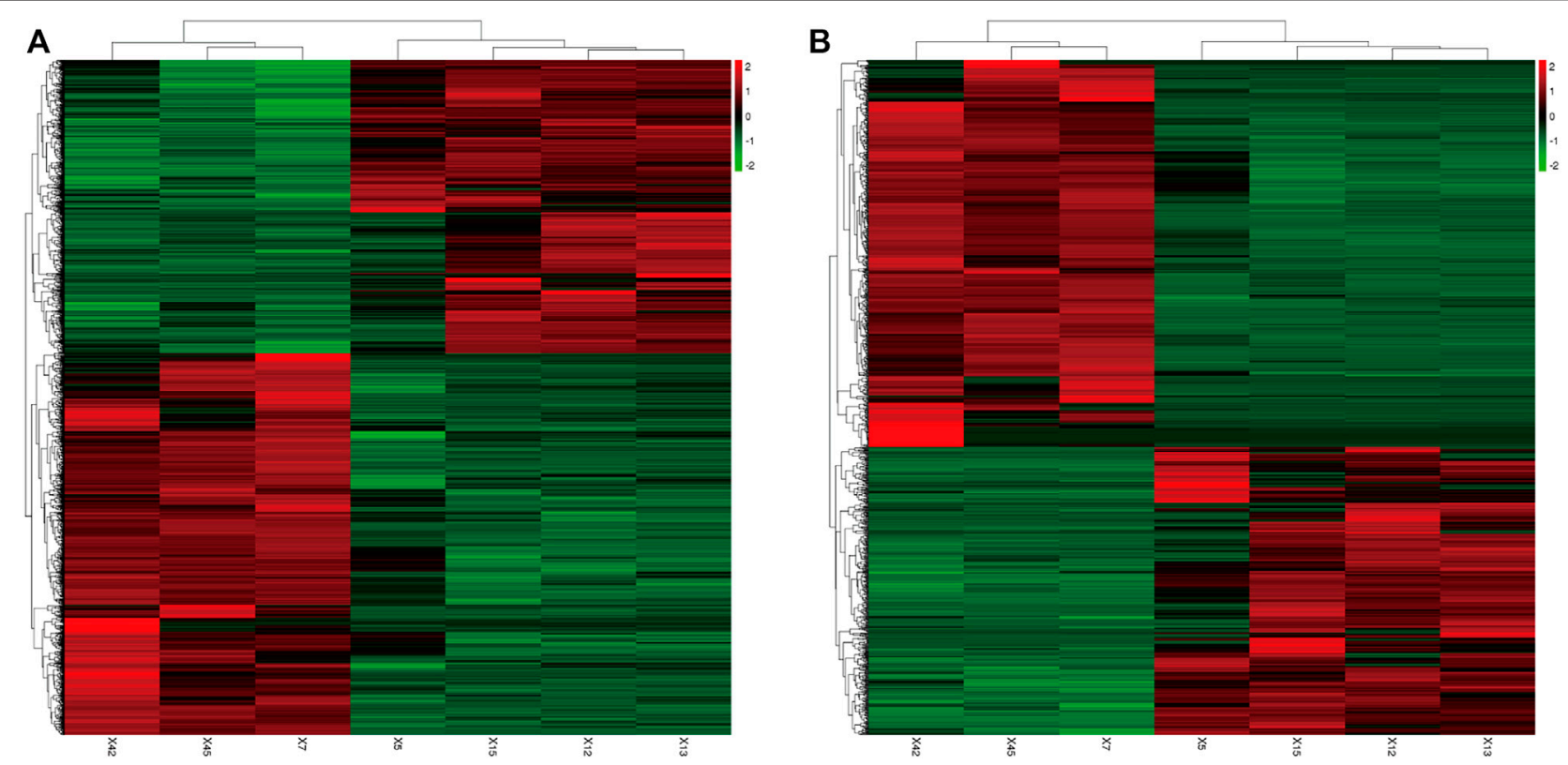

FIGURE 4 | Differential Gene Expressions (Heat map). (A): Differentially expressed mRNAs. (B): Differentially expressed IncRNAs. X5, X12, X13, X15: Asthma group. X7, X42, X45: Control group. Green: down-regulated; Red: up-regulated.

As shown in Figure 4, red color indicated that the genes were upregulated, while green color was down-regulated. The darker the color was, the higher the multiples of up-regulation or downregulation it indicated. In addition, the top 50 up and downregulated lncRNAs (Supplementary Tables S4, S5) as well as top 50 mRNAs (Supplementary Tables S6, S7) were screened out. Scatter plots and volcano plots were generated to display the differences between the two groups (Figure 5).

\section{Comparative Analysis of Differentially Expressed IncRNAs and mRNAs}

The transcript length, number of exons, and expression levels of differentially expressed lncRNAs and mRNAs were compared and analyzed. The length of the lncRNA transcripts was similar to that of mRNA. The proportion of lncRNA transcripts with $\leq 3$ exons was significantly greater than that of mRNA. The average FPKM expression of each lncRNA and mRNA transcript was used to generate a box plot of $\log 10(\mathrm{FPKM}+1)$. The expression levels of lncRNA were similar to those of mRNA but were more uniform in the expression range of 0-2.5 FPKM (Figure 6). This suggests that the lncRNAs identified in the present study conform to the general characteristics of lncRNAs.

\section{GO and KEGG Analysis of Differentially Expressed Genes}

We next performed GO analysis using the DEGs identified by sequencing. Of the DEGs, 2,290 mapped on to 57 functional GO terms. Among these, 26 were related to biological processes, with cellular process (GO:0009987) being the most frequent, 17 were related to cellular component, mainly cell (GO:0005623) and cell part (GO:0044464), and 14 were related to molecular functions, mainly binding (GO:0005488) (Figure 7A).

We next performed GO enrichment analysis on the differentially expressed genes and selected the top 30 significantly enriched GO entries. As shown in Figure $\mathbf{7 B}$, the enriched biological processes included nuclear-transcribed mRNA catabolic process (GO:0000956), establishment of protein localization to endoplasmic reticulum (GO:0072599), endoplasmic reticulum protein targeting process (protein targeting to ER, GO:0006612), and co-translational protein targeting to the membrane (GO:0006613). The most enriched cellular component was cytosolic ribosomes (GO:0022626) (see Supplementary Table S8).

We next performed KEGG pathway enrichment analysis on the differentially expressed genes between the two groups, and identified the top 30 enriched KEGG pathways. As shown in Figures $\mathbf{8 A , B}$, pathways related to the immune system, signal transduction, translation, cancers: overview, global and overview maps, cellular community, and environmental information processing were enriched, among which signal transduction in environmental information processing were the most enriched. Among the top 30 enriched signaling pathways, the ribosome (hsa03010) contained the highest number of DEGs (see Supplementary Table S9).

\section{Differentially Regulated IncRNA and IncRNA-mRNA Co-expression Network}

We filtered the top 50 upregulated lncRNAs (Supplementary Table S4) and the top 50 downregulated lncRNAs (Supplementary Table S5) in the asthma group compared to in the healthy control group. Based on the KEGG pathway 
A
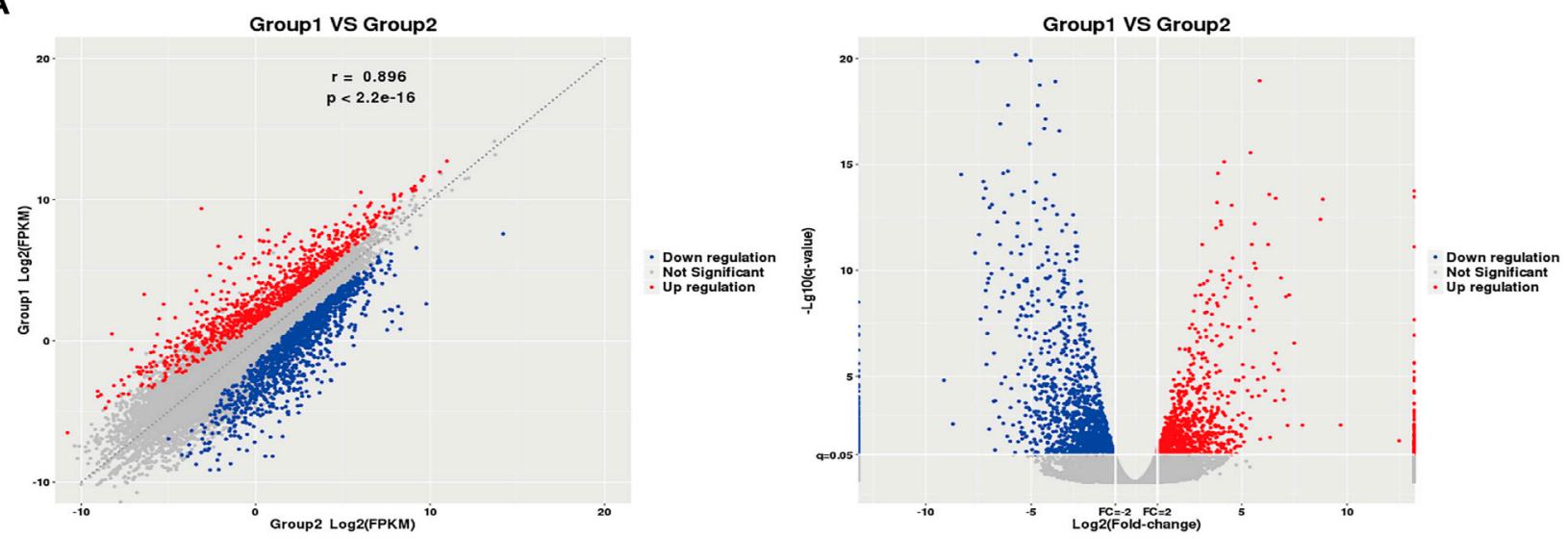

B
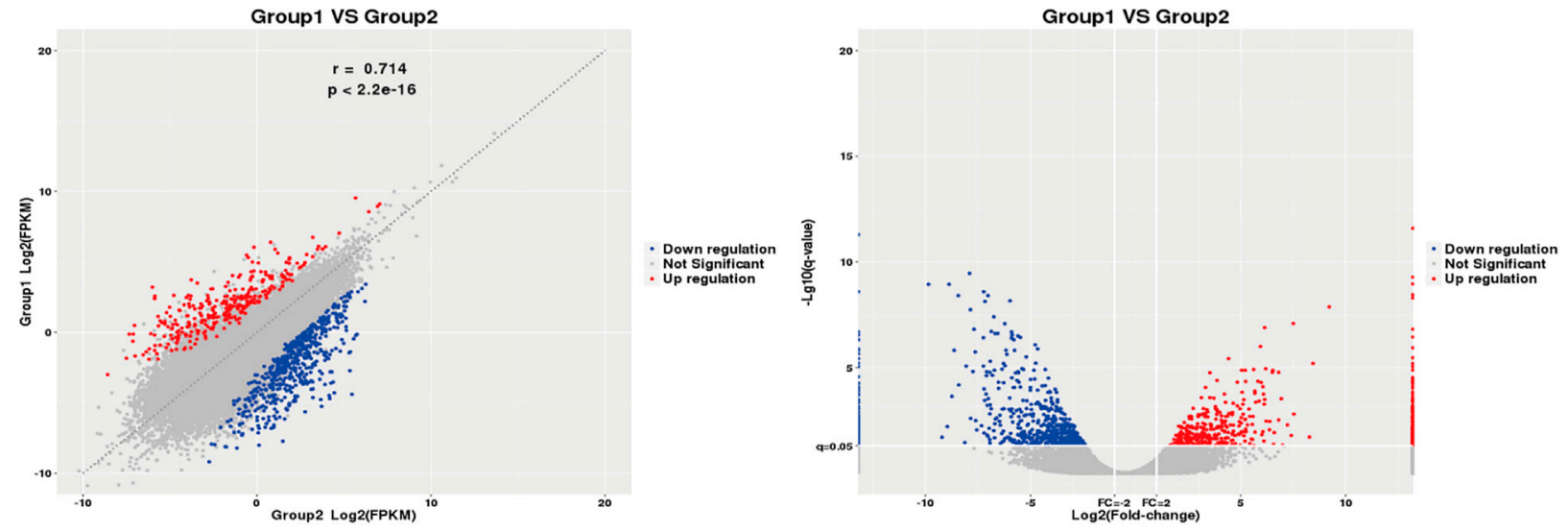

FIGURE 5 | Differential Gene Expressions (scatter and volcano plots). (A): Differentially expressed mRNAs. (B): Differentially expressed IncRNAs. X5, X12, X13, X15: Asthma group. X7, X42, X45: Control group. Blue: down-regulated; Red: up-regulated. FPKM: Fragments Per kilobase of exon model per Million mapped reads.

enrichment analysis, we selected differentially expressed mRNAs in 25 signaling pathways (Table 6) that were related to asthma in the literature. According to lncRNA-mRNA co-expression analysis (Figure 9), the top $10 \mathrm{lncRNAs}$ in the co-expression network were selected, of which seven were down-regulated and three were up-regulated (Table 7). Among these, IDs starting with MSTRG are novel lncRNAs, IDs starting with NON are known lncRNAs in the database, and IDs starting with ENS are known lncRNAs in the Ensembl database.

\section{Verification of Differentially Expressed IncRNAs}

We performed qPCR on peripheral blood samples from four asthma patients and three healthy subjects that were previously sequenced to verify the sequencing results. The gene sequence of lncRNA NONHSAT151355.1 overlaps completely with the mRNA sequence LAPTM5, and the gene sequence of lncRNA NONHSAT220907.1 overlaps completely with the mRNA sequence of UBQLN1, so specific primers could not be designed for these lncRNAs. Therefore, we designed specific primer sets for the remaining eight asthma-associated lncRNAs. As shown in Figure 10, the relative expression of lncRNA ENST00000615535 in the asthma group was lower than that in the healthy control group, which was inconsistent with the sequencing results, but this difference was not statistically significant $(p=0.535)$. The expression levels of the other 7 lncRNAs in the asthma group were all lower than in the healthy control group, which was consistent with the sequencing results, and the differences were statistically significant.

To further verify that the identified lncRNAs were an asthmaspecific signature, peripheral blood samples were obtained from three asthma patients and three healthy subjects that were randomly selected from the remaining subjects that were not included in the sequencing dataset. qPCR analysis of these expanded samples revealed that the expression trends of two of the lncRNAs (NONHSAT163272.1 and NONHSAT190964.1) were consistent with the trends observed in the sequencing dataset, and these differences were statistically significant (both $p<0.05$ ) (Figure 11). Although the remaining target lncRNAs had no statistically significant differences in the expanded sample set between the asthma and healthy control groups, their 


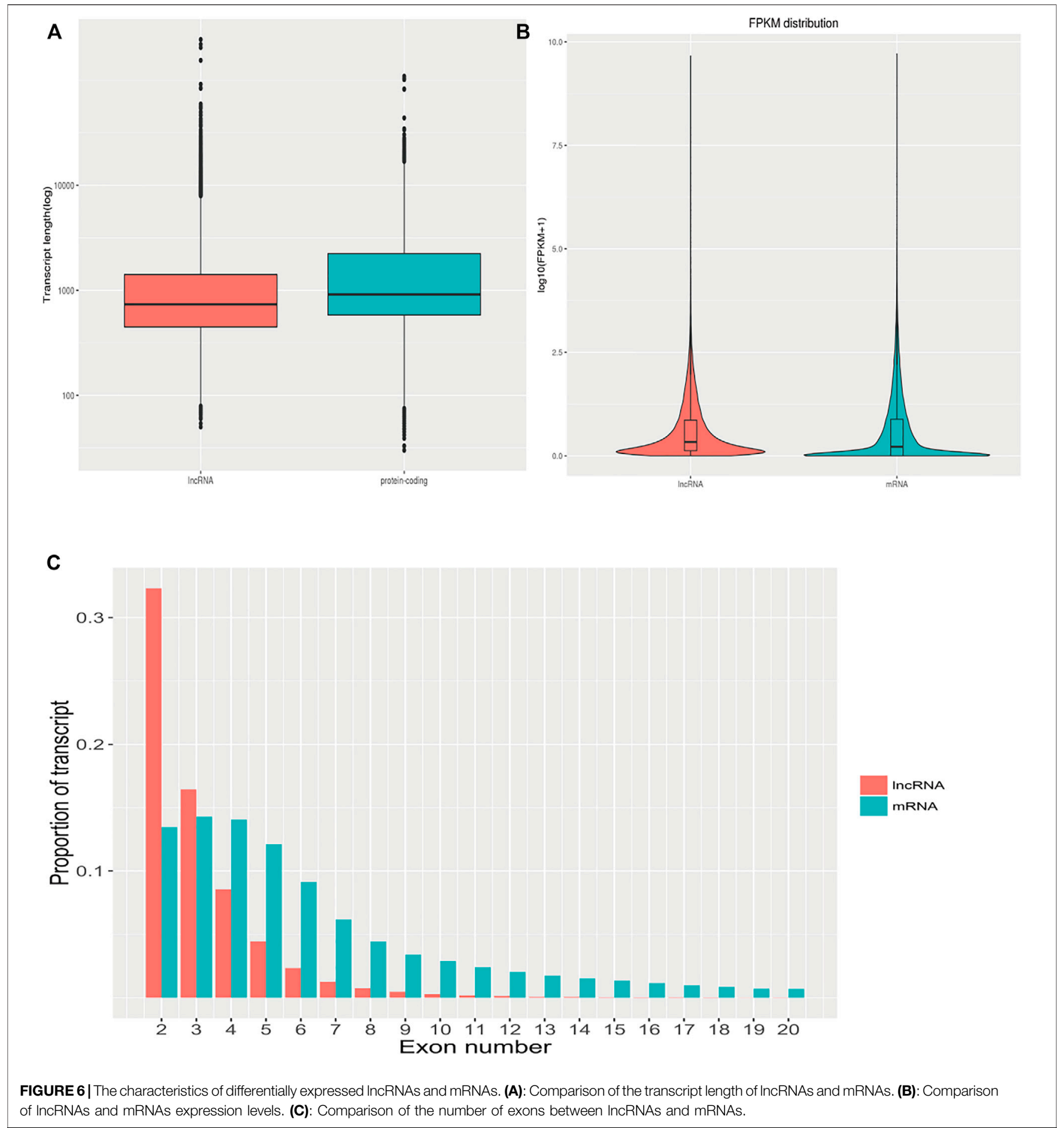

expression trend was consistent with those of the sequenced samples.

Next, we compared the expression of the 7 identified lncRNAs from the present study with their expression in four public databases in the Gene Expression Omnibus (GEO). Among these four databases, two of them performed lncRNA sequencing of whole blood from asthma patients (GSE106230, https://www.ncbi.nlm.nih.gov/geo/query/acc.cgi?acc=
GSE106230, adults; GSE117038, https:/www.ncbi.nlm.nih.gov/ geo/query/acc.cgi?acc=GSE117038, adults), and the other two used microarrays to detect the expression of lncRNAs in the peripheral blood mononuclear cells (PBMCs) of asthmatic patients (GSE165934, https://www.ncbi.nlm. nih.gov/geo/query/acc.cgi?acc=GSE165934, adults; GSE143192, https://www.ncbi.nlm.nih.gov/geo/query/acc. cgi?acc=GSE143192, children). 


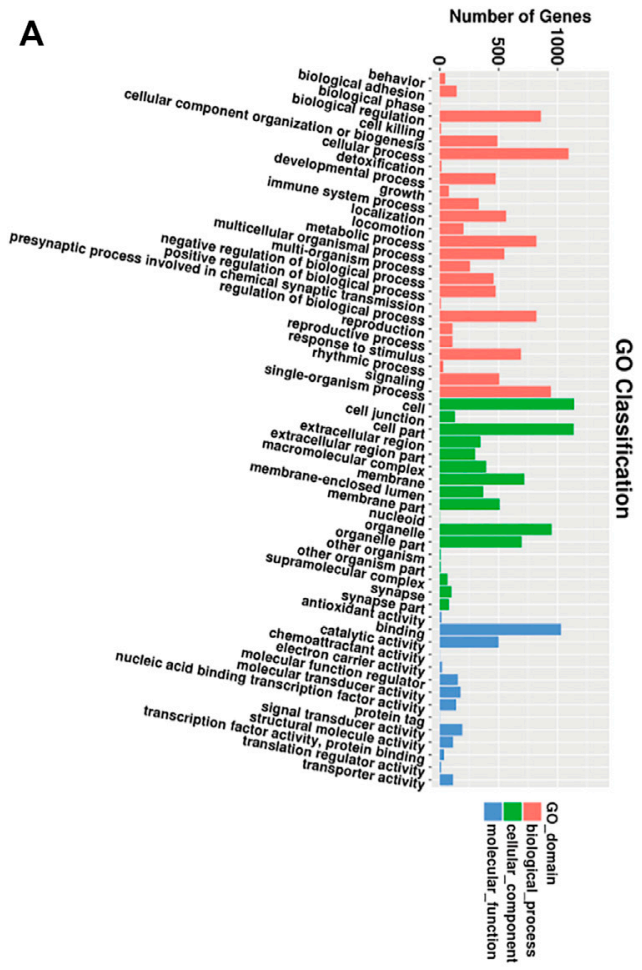

B

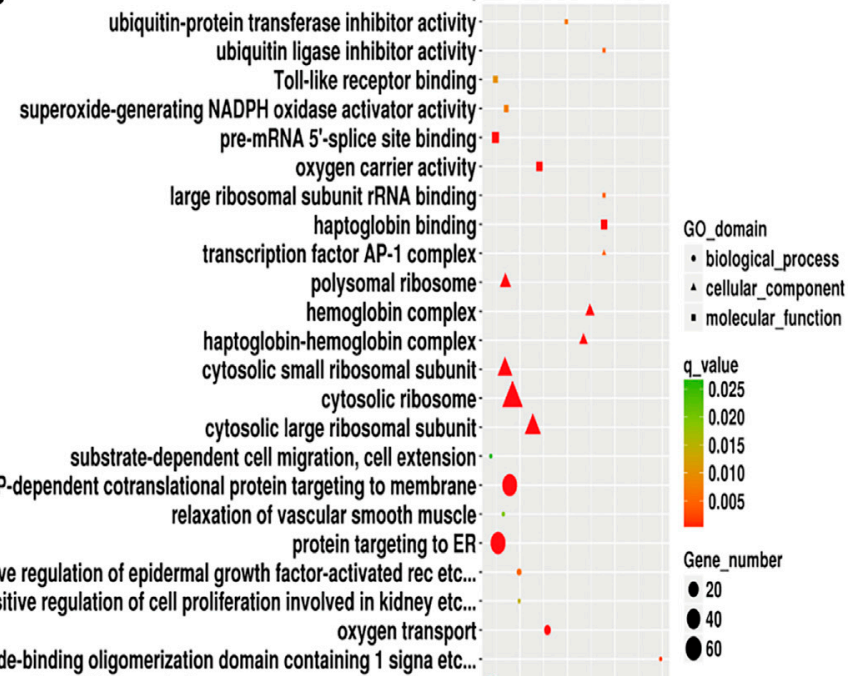
nucleotide-binding oligomerization domain containing 1 signa etc.. neuron projection maintenance negative regulation of ubiquitin protein ligase activity mitochondrial electron transport, cytochrome c to oxygen-• formation of quadruple SL/U4/U5/U6 snRNP. establishment of protein localization to endoplasmic reticul etc... . cotranslational protein targeting to membrane.
Top 30 of GO Enrichment

- biological_process - molecular_function

value

0.015

0.010

Gene_number

20
40
60

FIGURE 7 | GO analysis of differentially expressed genes. (A): GO function classification map of differentially expressed genes. (B): Scatter plot of GO enrichment of top 30 differentially expressed genes.
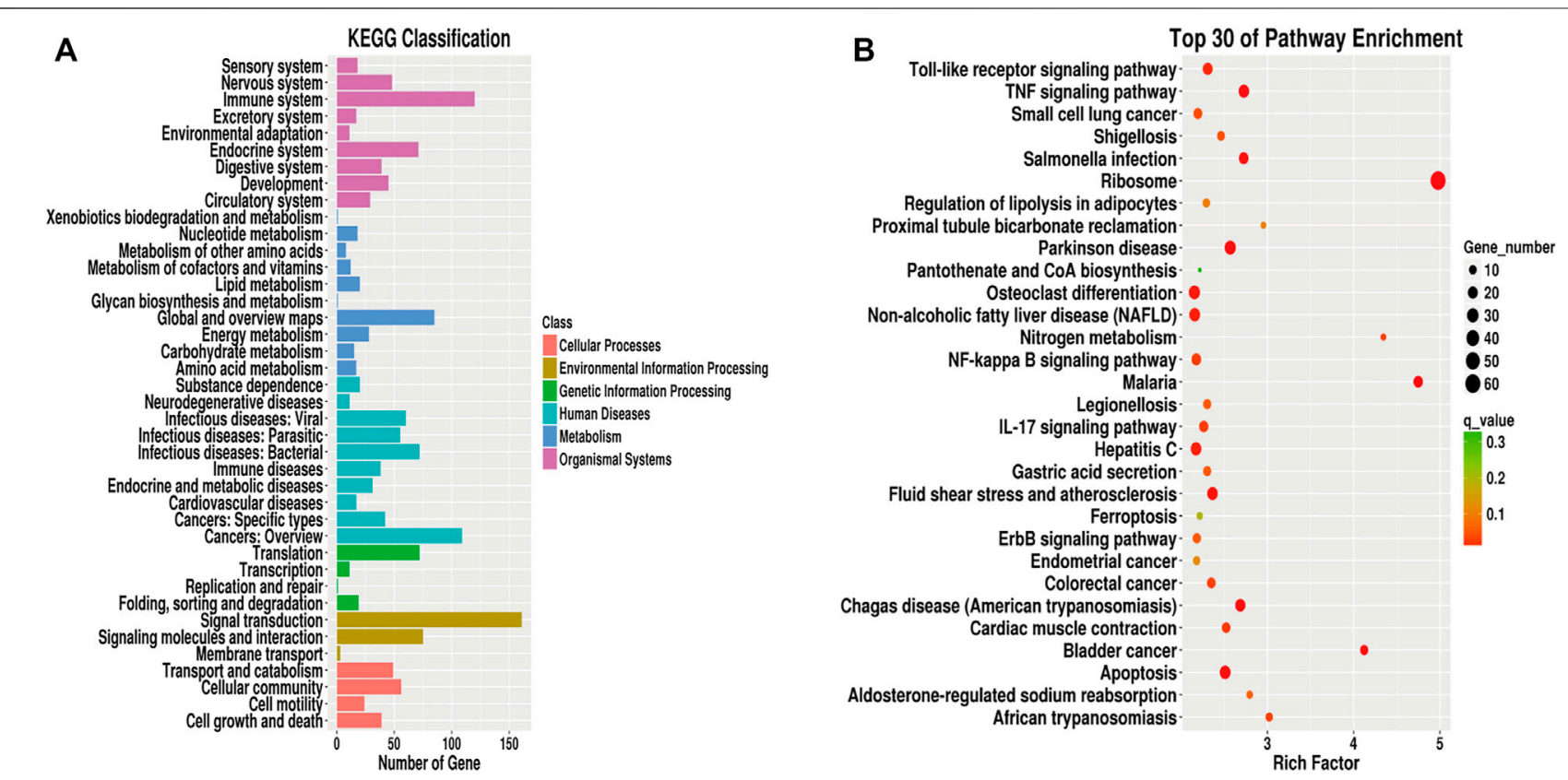

FIGURE 8| KEGG analysis of differentially expressed genes. (A): KEGG pathway classification map of differentially expressed genes. (B): KEGG enrichment scatter plot of differentially expressed genes. 
TABLE 6 | Asthma-related KEGG enrichment pathway.

\begin{tabular}{|c|c|c|c|}
\hline Pathway_id & Pathway & Diff_Genes & Rich factor \\
\hline hsa01100 & Metabolic pathways & 84 & 0.902 \\
\hline hsa03010 & Ribosome & 60 & 4.979 \\
\hline hsa04060 & Cytokine-cytokine receptor interaction & 46 & 2.016 \\
\hline hsa04151 & PI3K-Akt signaling pathway & 43 & 1.694 \\
\hline hsa04010 & MAPK signaling pathway & 38 & 1.691 \\
\hline hsa04062 & Chemokine signaling pathway & 28 & 1.897 \\
\hline hsa04210 & Apoptosis & 26 & 2.510 \\
\hline hsa04668 & TNF signaling pathway & 24 & 2.727 \\
\hline hsa00190 & Oxidative phosphorylation & 23 & 2.150 \\
\hline hsa04024 & cAMP signaling pathway & 23 & 1.530 \\
\hline hsa04621 & NOD-like receptor signaling pathway & 21 & 1.385 \\
\hline hsa04620 & Toll-like receptor signaling pathway & 20 & 2.308 \\
\hline hsa04514 & Cell adhesion molecules (CAMs) & 20 & 1.098 \\
\hline hsa04064 & NF-kappa B signaling pathway & 19 & 2.175 \\
\hline hsa04630 & JAK-STAT signaling pathway & 18 & 1.592 \\
\hline hsa04657 & IL-17 signaling pathway & 17 & 2.262 \\
\hline hsa04150 & mTOR signaling pathway & 16 & 1.343 \\
\hline hsa04659 & Th17 cell differentiation & 12 & 0.909 \\
\hline hsa04650 & Natural killer cell mediated cytotoxicity & 11 & 0.339 \\
\hline hsa04660 & T cell receptor signaling pathway & 9 & 1.156 \\
\hline hsa04662 & B cell receptor signaling pathway & 8 & 1.515 \\
\hline hsa03320 & PPAR signaling pathway & 8 & 1.477 \\
\hline hsa04750 & Inflammatory mediator regulation of TRP channels & 8 & 1.158 \\
\hline hsa04152 & AMPK signaling pathway & 8 & 0.916 \\
\hline hsa04310 & Wnt signaling pathway & 7 & 0.601 \\
\hline
\end{tabular}

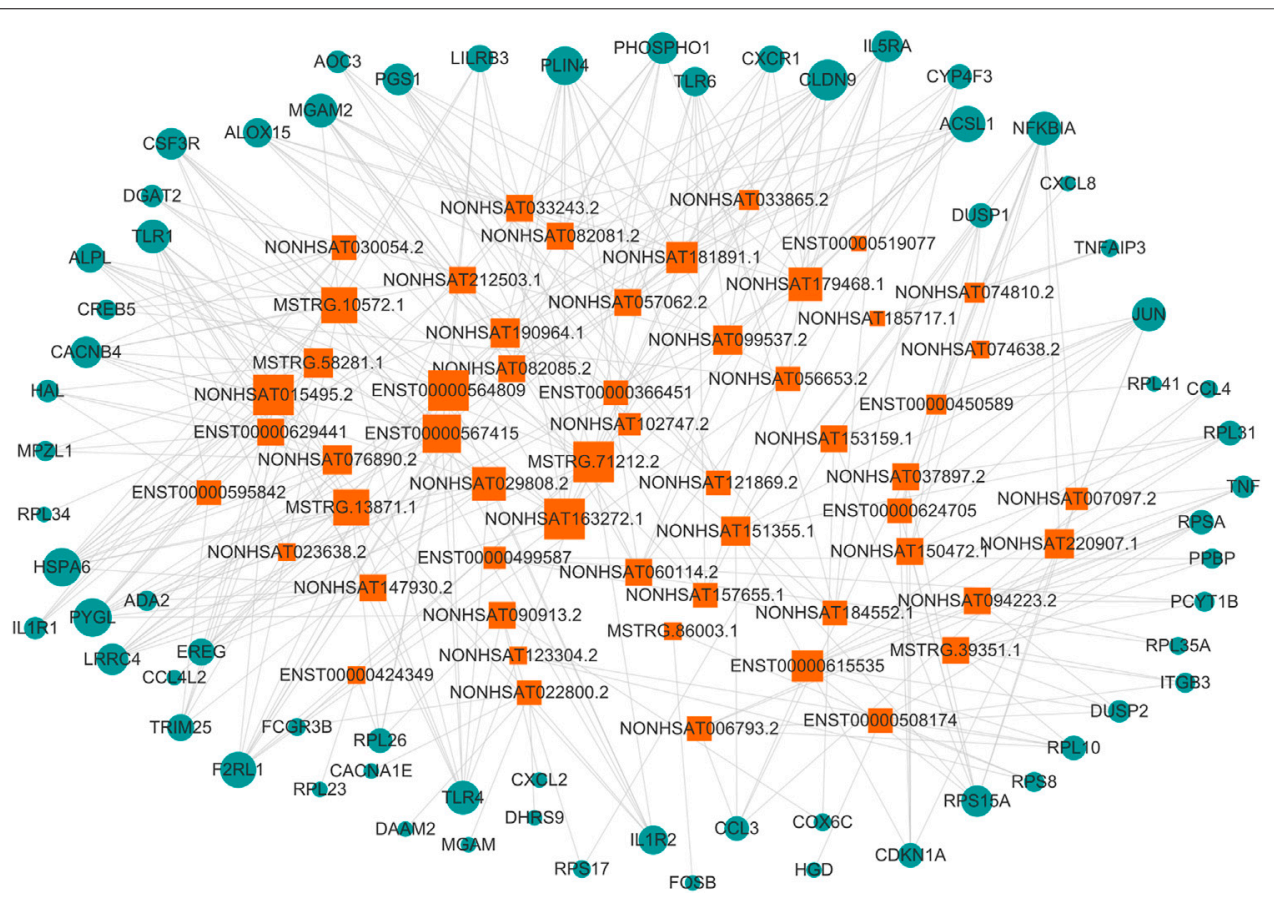

FIGURE 9 | IncRNA-mRNA co-expression network. The circle represents mRNA, the square represents IncRNA, and the line between the circle and the square represents the regulatory relationship between genes. The relationship between a gene and other genes in the network is represented by the "degree value", the greater the degree value, the larger the area of the circle and the square.

For the original data from GSE106230 and GSE117038, we performed differential lncRNA analysis (Supplementary Figure S1) and then matched these results with the 7 lncRNAs from the present study. For those IncRNAs with no matches in the differential lncRNA analysis results, we matched them to the entire GSE106230 and GSE117038 database. For the original data 
TABLE 7 | Top 10 IncRNAs with degree value.

IncRNA_id IncRNA name

\section{NONHSAT015495.2}

MSTRG.71212.2

NONHSAT163272.1

ENST00000564809

NONHSAT181891.1

ENST00000615535

NONHSAT151355.1

NONHSAT190964.1

NONHSAT220907.1

NONHSAT076890.2

Inc-FAS-2-2_dup1
-
-
Inc-KLHL36-1-1
XLOC_001496
-
m130127_174,513_00126_c100
Inc-DSCR8-3-1_dup1
m121212_111,138_00126_c100
Inc-CXCR2-1-5_dup1

Up/down degree

down 12

down 12

down 12

down 12

down 9

up 8

up 7

down 7

up 7

down 7

from GSE165934 and GSE143192, due to the different samples and detection methods used, we directly performed the comparison. As shown in Figure 12, in the GSE106230 and GSE117038 datasets contained five of the targeting differentially expressed lncRNAs from the present study (ENST00000564809, NONHSAT015495.2,

NONHSAT076890.2, NONHSAT181891.1, and NONHSAT190964.1). In the GSE106230 database, the FPKM values for the lncRNAs ENST00000564809, NONHSAT015495.2, NONHSAT181891.1, and NONHSAT190964.1 showed a downward trend compared with the healthy group, which is consistent with our results, although the differences between the case and control groups
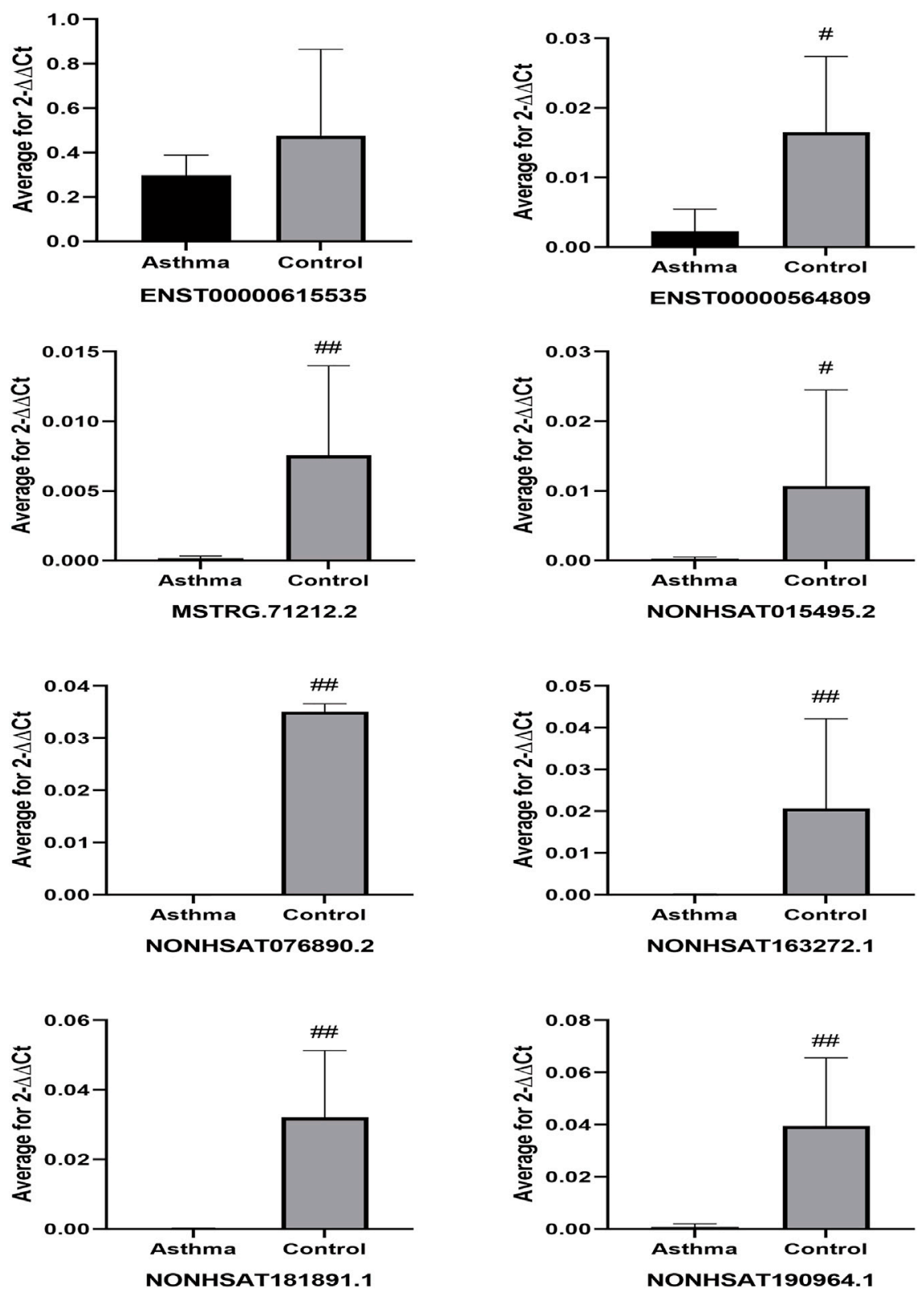

FIGURE 10 | Differential expression of IncRNAs in sequencing samples. ${ }^{\#} p<0.05,{ }^{\# \#} p<0.01$ vs Asthma group. 

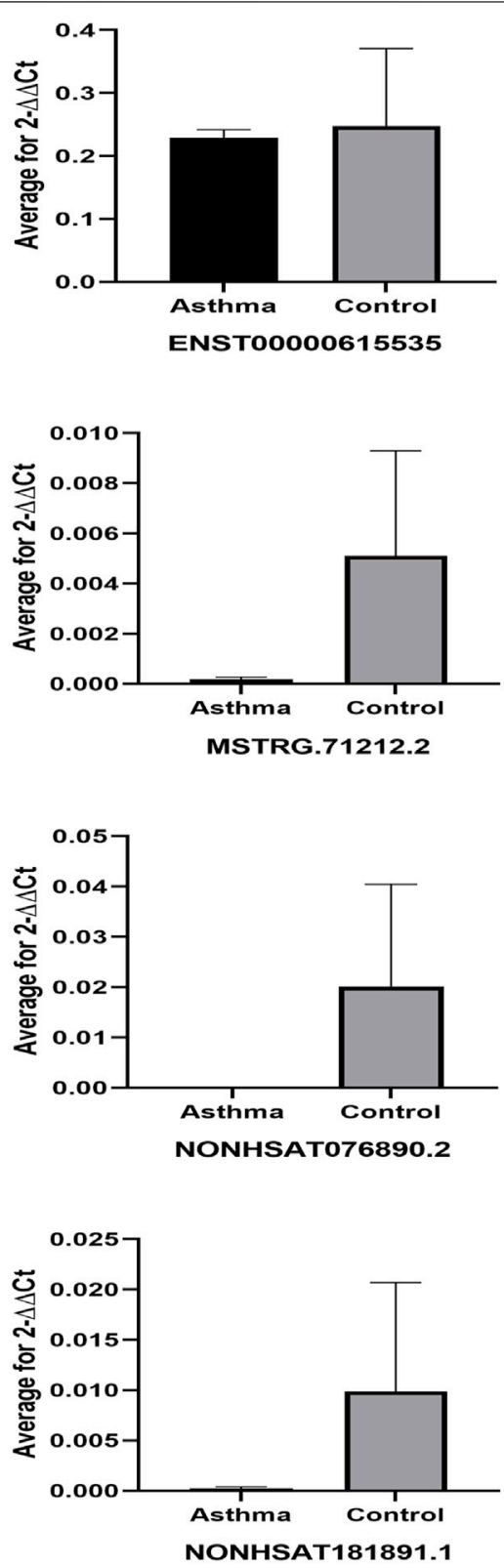
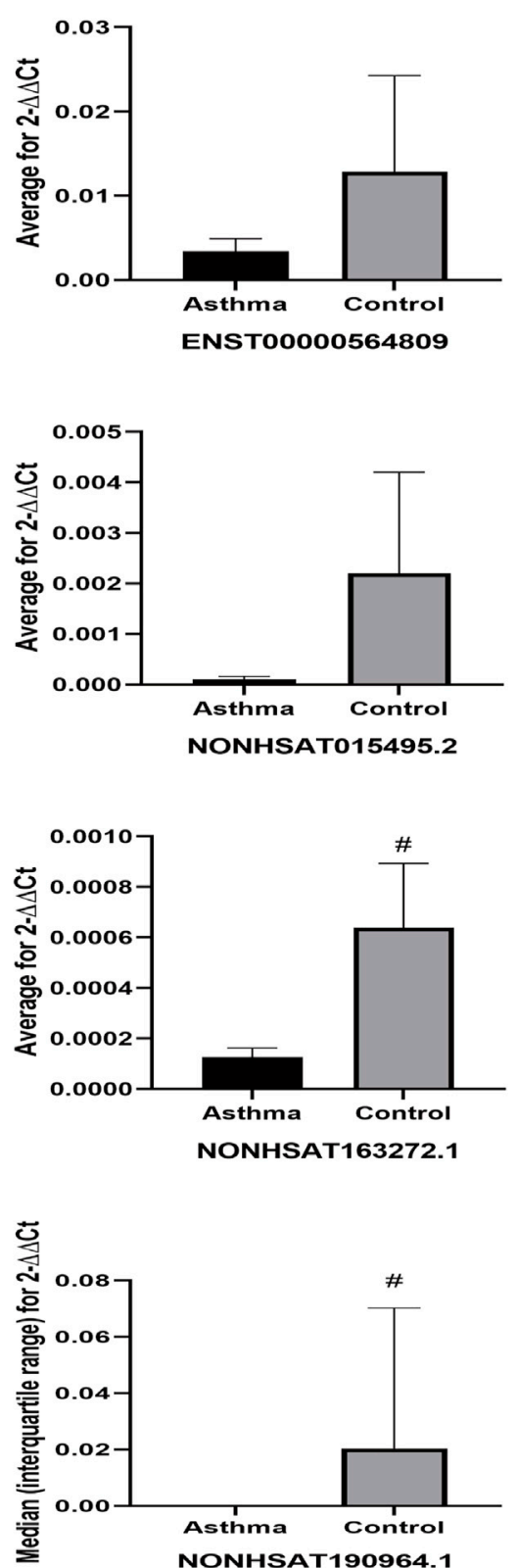

FIGURE 11 | Differential expression of IncRNAs in expanding samples. \#p < 0.05 vs Asthma group.

were not statistically significant (Figure 12A). In the GSE117038 database, we excluded samples from patients with neutrophilic asthma (the patients we included were eosinophil-dominated). As shown in Figure 12B, compared with the healthy group, these five lncRNAs were not statistically different in the asthmatic group in this dataset. Four of the lncRNAs (ENST00000564809, NONHSAT015495.2, NONHSAT076890.2, and NONHSAT190964.1) were slightly upregulated in the asthma group compared with in healthy subjects, which contradicts our results. There were no matches between the seven identified lncRNAs from the present study in the GSE165934 dataset. In the GSE143192 database, the FPKM values of ENST00000564809 and NONHSAT015495.2 were slightly increased in children with asthma compared with in healthy children (Figure 12C).

\section{DISCUSSION}

Asthma is a chronic inflammatory disease of the lower respiratory tract that is characterized by reversible airflow obstruction and airway remodeling (Bousquet et al., 2000). Early research on the mechanisms of asthma mainly focused on T helper (Th) cells. It is generally thought that a Th1/Th2 imbalance (Th1 inhibition and Th2 hyperfunction) is the main cause of airway 
A
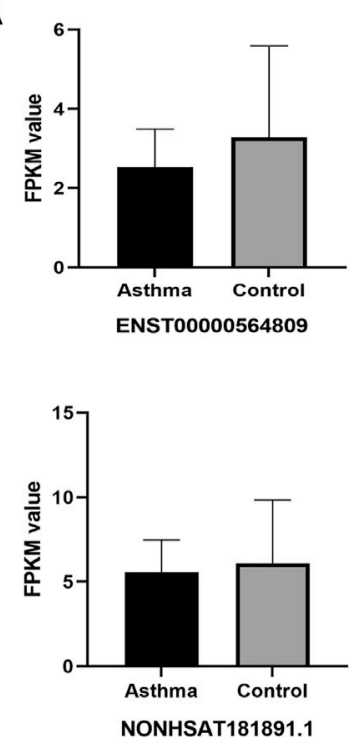

B
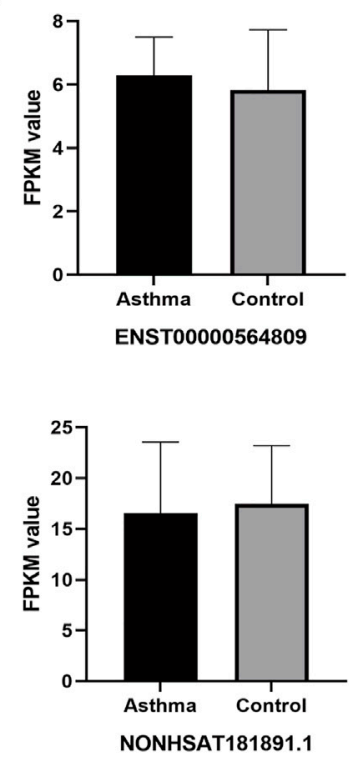

C

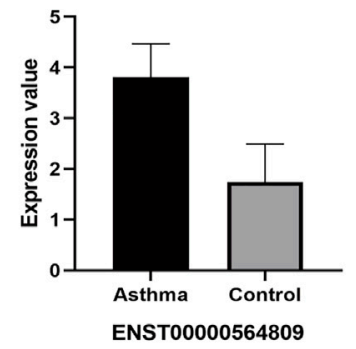

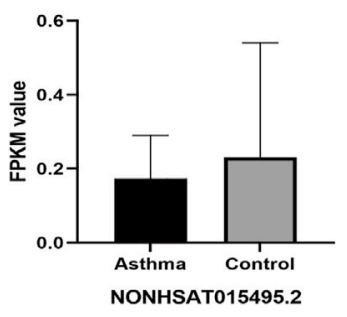
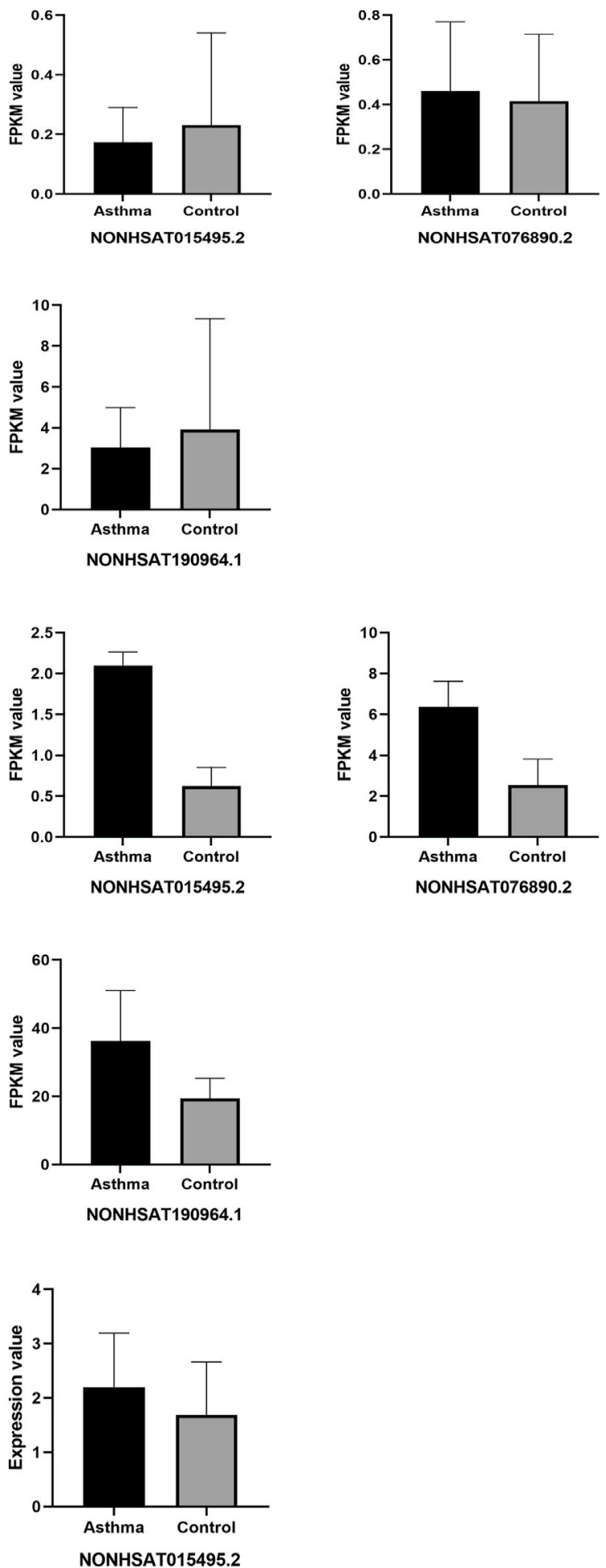

FIGURE 12|Differential expression of target IncRNAs in public databases. (A): Each target of differential IncRNAs in the GSE106230 database; (B): Each target of differential IncRNAs in the GSE117038 database; (C): Each target of differential IncRNAs in the GSE143192 database. 
hyperresponsiveness and chronic inflammation in asthma (Ngoc et al., 2005). Recent research has advanced our understanding of the pathogenesis of asthma, and suggests that a Treg/Th17 imbalance is as important as a Th1/Th2 imbalance, and is closely related to the progression and severity of asthma (Wang et al., 2018). However, this cannot fully explain the pathogenesis of asthma. For example, under certain circumstances, the enhancement of Th1 activity can aggravate asthma symptoms, rather than acting as a protective factor (Zou et al., 2018). With the development of advanced technology for studying epigenetics and transcriptomics in recent years, there is growing evidence that non-coding RNAs are involved in the process of asthma pathology, and play a crucial role in pre- and post-transcriptional regulation (Ghafouri-Fard et al., 2020), such as lncRNAs can regulate the proliferation, differentiation, and induction of immune/structural cells, and are closely related to asthma (Austin et al., 2017; Zhu et al., 2019; Liu et al., 2020).

Although there have been clinical trials to detect the differential expression of lncRNAs in the peripheral blood of patients with asthma (Gál et al., 2020; Zheng et al., 2020), there are many lncRNAs that have not been annotated. Finding other lncRNAs that may play a key role in asthma is therefore important, and may contribute to a better understanding and treatment of asthma. Therefore, in the present study, we used second-generation high-throughput sequencing technology to perform whole-transcriptome sequencing in asthma patients and healthy subjects, and identified the differentially expressed mRNAs and lncRNAs. We identified a total of 2,290 differentially expressed mRNAs and 1,137 differentially expressed lncRNAs in asthma patients compared with in healthy subjects. Among these, there were 994 upregulated and 1,296 downregulated mRNAs, and 485 upregulated and 652 downregulated differential lncRNAs. We conducted a comparative analysis of the identified lncRNAs and mRNAs to further verify that the lncRNAs were lncRNAs. Then, we performed GO and KEGG functional analysis of the differentially expressed genes. The GO and KEGG enrichment results showed that the cytosolic ribosome (GO:0022626) and ribosome (hsa03010) were highly enriched in the DEGs. This may be because mRNA carries the code from the DNA in the nucleus to the ribosome in the cytoplasm, and protein synthesis is often regulated by structured mRNAs that interact with ribosomes (Boehringer and Ban, 2007). For example, gene expression can be regulated by structural elements in the $5^{\prime}$ untranslated region of mRNA at the initial level of protein biosynthesis, and these folded mRNA fragments may bind to ribosomes, preventing translation (Marzi et al., 2007). We selected the top 50 up-regulated and top 50 down-regulated $\operatorname{lncRNAs}$ and identified the top 25 pathways related to asthma. Following co-expression analysis, we generated a lncRNA-mRNA co-expression network and selected the top 10 differentially expressed lncRNAs according to the descending order of degree value. Among these 10 differentially expressed lncRNAs, NONHSAT015495.2, MSTRG.71212.2, NONHSAT163272.1, ENST00000564809, NONHSAT181891.1, NONHSAT190964.1, and NONHSAT076890.2, were downregulated in asthma patients, and ENST00000615535, NONHSAT151355.1, and
NONHSAT220907.1 were upregulated in asthma patients. We used qPCR to verify the sequencing results for these 10 lncRNAs. Except for the decreased expression of ENST00000615535 in asthma patients, which was inconsistent with the sequencing results, the expression of the remaining seven lncRNAs (NONHSAT015495.2, MSTRG.71212.2, NONHSAT163272.1, ENST00000564809, NONHSAT181891.1, NONHSAT190964.1, and NONHSAT076890.2) was relatively lower in asthma patients than in healthy subjects, and thus confirmed the sequencing results. To verify that these lncRNAs are an asthma-specific signature, we measured their expression by qPCR in new subjects and cases randomly selected from the remaining samples that were not sequenced, and the expression of two of the lncRNAs (NONHSAT163272.1 and NONHSAT190964.1) were consistent with the sequencing dataset.

In addition, we investigated their expression in datasets from the GEO database. The FPKM value trends of four of the differentially expressed lncRNAs identified in the present study (ENST00000564809, NONHSAT015495.2, NONHSAT181891.1, and NONHSAT190964.1) in the GSE106230 dataset were consistent with our results, although the differences were not statistically significant in that dataset. However, the FPKM value trends of the four differentially expressed lncRNAs (ENST00000564809, NONHSAT015495.2, NONHSAT076890.2, NONHSAT190964.1) in the GSE117038 database, and two lncRNAs (ENST00000564809, NONHSAT015495.2) in the GSE143192 database disagreed with the results of the present study. It is worth noting that samples used in the GSE106230 and GSE117038 databases (whole blood samples from adults), and the PBMC samples used in the GSE143192 database (children) did not match the sample type used in the present study, perhaps limiting the utility of this comparison.

The present study had some limitations. As shown in Table 3, there were no statistically significant differences observed for Th2 cytokines, including IL-4, IL-5, and IL-13, between the asthma and healthy control subjects in the present study. This may be due to the choice of peripheral blood as detection samples, and the regular treatment of low dose ICS/LABA for asthmatic patients before the recruitment. It is agreed that the main pathology of asthma patients is in the airways, and Bronchoalveolar Lavage Fluid (BALF) may therefore be a better sample type for the detection of asthma-derived inflammatory factors. However, fiberoptic bronchoscopy is an invasive procedure and not routinely performed in asthma patients, which made the collection of BALF difficult. As venous blood was easy to collect and had little harm to participants, it was used to detect the cytokine levels instead; on the other hand, the regular use of ICS/LABA in asthma patients might to some degree alleviate the inflammatory status so that rare differences of inflammatory cytokines were found between asthma patients and healthy subjects. Due to the small sample size of the present study, the proposed asthma-specific marker lncRNAs could not be confirmed as biomarkers for asthma. To address this, multicentered clinical study with a larger sample size should be undertaken. In addition, the composition of lncRNAs present in the peripheral blood may be affected by many factors, and we did not take the atopic status and some allergy-related co- 
morbidities into consideration during the participants recruitment, and to some degree this negligence potentially affect the gene expressions in PBMCs in whole blood. Animal models could be used to measure their expression in the lung tissue and BALF specifically, which may provide more compelling evidence that they are directly related to the pathology of asthma. The regulatory effect of these lncRNAs in immune/structural cells should also be confirmed by in vitro studies, which could also provide much-needed functional annotation for these potentially important lncRNAs.

In conclusion, we have identified a panel of putative asthmaassociated lncRNAs (NONHSAT015495.2, MSTRG.71212.2, NONHSAT163272.1, ENST00000564809, NONHSAT181891.1, NONHSAT190964.1, and NONHSAT076890.2), which may play a role in the pathogenesis of asthma, and may be useful as diagnostic or prognostic biomarkers in the clinical setting.

\section{DATA AVAILABILITY STATEMENT}

The datasets presented in this study can be found in online repositories. https://www.ncbi.nlm.nih.gov/geo/query/acc.cgi?acc=GSE195599.

\section{ETHICS STATEMENT}

The studies involving human participants were reviewed and approved by the Ethical Review Committee of Huashan Hospital

\section{REFERENCES}

Austin, P. J., Tsitsiou, E., Boardman, C., Jones, S. W., Lindsay, M. A., Adcock, I. M., et al. (2017). Transcriptional Profiling Identifies the Long Noncoding RNA Plasmacytoma Variant Translocation (PVT1) as a Novel Regulator of the Asthmatic Phenotype in Human Airway Smooth Muscle. J. Allergy Clin. Immunol. 139 (3), 780-789. doi:10.1016/j.jaci.2016.06.014

Boehringer, D., and Ban, N. (2007). Trapping the Ribosome to Control Gene Expression. Cell 130 (6), 983-985. doi:10.1016/j.cell.2007.09.002

Bousquet, J., Jeffery, P. K., Busse, W. W., Johnson, M., and Vignola, A. M. (2000). Asthma. From Bronchoconstriction to Airways Inflammation and Remodeling. Am. J. Respir. Crit. Care Med. 161 (5), 1720-1745. doi:10.1164/ajrccm.161.5. 9903102

Chen, H., and Shan, G. (2020). The Physiological Function of Long-Noncoding RNAs. Noncoding RNA Res. 5 (4), 178-184. doi:10.1016/j.ncrna.2020.09.003

Côté, A., Godbout, K., and Boulet, L. P. (2020). The Management of Severe Asthma in 2020. Biochem. Pharmacol. 179, 114112. doi:10.1016/j.bcp.2020.114112

Fahy, J. V. (2015). Type 2 Inflammation in Asthma-Ppresent in Most, Absent in many. Nat. Rev. Immunol. 15 (1), 57-65. doi:10.1038/nri3786

Gál, Z., Gézsi, A., Semsei, Á. F., Nagy, A., Sultész, M., Csoma, Z., et al. (2020). Investigation of Circulating IncRNAs as Potential Biomarkers in Chronic Respiratory Diseases. J. Transl Med. 18 (1), 422. doi:10.1186/s12967-020-02581-9

Ghafouri-Fard, S., Shoorei, H., Taheri, M., and Sanak, M. (2020). Emerging Role of Non-coding RNAs in Allergic Disorders. Biomed. Pharmacother. 130, 110615. doi:10.1016/j.biopha.2020.110615

Hammad, H., and Lambrecht, B. N. (2021). The Basic Immunology of Asthma. Cell 184 (6), 1469-1485. doi:10.1016/j.cell.2021.02.016

Kudo, M., Melton, A. C., Chen, C., Engler, M. B., Huang, K. E., Ren, X., et al. (2012). IL-17A Produced by a $\beta$ T Cells Drives Airway Hyper-Responsiveness in Mice
Affiliated to Fudan University. The patients/participants provided their written informed consent to participate in this study.

\section{AUTHOR CONTRIBUTIONS}

CM, SW collected subjects, performed the statistical analysis and wrote the manuscript; YC, WT, TW reviewed and editing the manuscipt, FT, XZ collected the data; YW, JD conceived and planned the study and supervised the study.

\section{FUNDING}

This work was supported by the National Natural Science Foundation of China (Grant No. 82174495 and 82174170); The 3 years to accelerate the development of Chinese medicine in Shanghai, China (Grant No: ZY (2018-2020)FWTX-4016); Dong Jingcheng Expert Workstation of Yunnan Province.

\section{SUPPLEMENTARY MATERIAL}

The Supplementary Material for this article can be found online at: https://www.frontiersin.org/articles/10.3389/fphar.2022.834009/ full\#supplementary-material

and Enhances Mouse and Human Airway Smooth Muscle Contraction. Nat. Med. 18 (4), 547-554. doi:10.1038/nm.2684

Liang, Z., and Tang, F. (2020). The Potency of lncRNA MALAT1/miR-155/CTLA4 axis in Altering Th1/Th2 Balance of Asthma. Biosci. Rep. 40 (2), BSR20190397. doi:10.1042/bsr20190397

Liu, J. H., Li, C., Zhang, C. H., and Zhang, Z. H. (2020). LncRNA-CASC7 Enhances Corticosteroid Sensitivity via Inhibiting the PI3K/AKT Signaling Pathway by Targeting miR-21 in Severe Asthma. Pulmonology 26 (1), 18-26. doi:10.1016/j. pulmoe.2019.07.001

Lodde, V., Murgia, G., Simula, E. R., Steri, M., Floris, M., and Idda, M. L. (2020). Long Noncoding RNAs and Circular RNAs in Autoimmune Diseases. Biomolecules 10 (7), 1044. doi:10.3390/biom10071044

Marzi, S., Myasnikov, A. G., Serganov, A., Ehresmann, C., Romby, P., Yusupov, M., et al. (2007). Structured mRNAs Regulate Translation Initiation by Binding to the Platform of the Ribosome. Cell 130 (6), 1019-1031. doi:10.1016/j.cell.2007.07.008

Ngoc, P. L., Ngoc, L. P., Gold, D. R., Tzianabos, A. O., Weiss, S. T., and Celedón, J. C. (2005). Cytokines, Allergy, and Asthma. Curr. Opin. Allergy Clin. Immunol. 5 (2), 161-166. doi:10.1097/01.all.0000162309.97480.45

Qiu, Y. Y., Wu, Y., Lin, M. J., Bian, T., Xiao, Y. L., and Qin, C. (2019). LncRNAMEG3 Functions as a Competing Endogenous RNA to Regulate Treg/Th17 Balance in Patients with Asthma by Targeting microRNA-17/ROR $\gamma$ t. Biomed. Pharmacother. 111, 386-394. doi:10.1016/j.biopha.2018.12.080

Vydzhak, O., Luke, B., and Schindler, N. (2020). Non-coding RNAs at the Eukaryotic rDNA Locus: RNA-DNA Hybrids and beyond. J. Mol. Biol. 432 (15), 4287-4304. doi:10.1016/j.jmb.2020.05.011

Wang, L., Wan, H., Tang, W., Ni, Y., Hou, X., Pan, L., et al. (2018). Critical Roles of Adenosine A2A Receptor in Regulating the Balance of Treg/Th17 Cells in Allergic Asthma. Clin. Respir. J. 12 (1), 149-157. doi:10.1111/crj.12503

Zheng, P., Huang, C., Leng, D., Sun, B., and Zhang, X. D. (2020). Transcriptome Analysis of Peripheral Whole Blood Identifies Crucial lncRNAs Implicated in 
Childhood Asthma. BMC Med. Genomics 13 (1), 136. doi:10.1186/s12920-02000785-y

Zhu, Y., Mao, D., Gao, W., Han, G., and Hu, H. (2019). Analysis of IncRNA Expression in Patients with Eosinophilic and Neutrophilic Asthma Focusing on LNC_000127. Front. Genet. 10, 141. doi:10.3389/fgene.2019.00141

Zou, X. L., Chen, Z. G., Zhang, T. T., Feng, D. Y., Li, H. T., and Yang, H. L. (2018). Th17/Treg Homeostasis, but Not Th1/Th2 Homeostasis, Is Implicated in Exacerbation of Human Bronchial Asthma. Ther. Clin. Risk Manag. 14, 1627-1636. doi:10.2147/tcrm.s172262

Conflict of Interest: The authors declare that the research was conducted in the absence of any commercial or financial relationships that could be construed as a potential conflict of interest.
Publisher's Note: All claims expressed in this article are solely those of the authors and do not necessarily represent those of their affiliated organizations, or those of the publisher, the editors and the reviewers. Any product that may be evaluated in this article, or claim that may be made by its manufacturer, is not guaranteed or endorsed by the publisher.

Copyright $\odot 2022$ Ma, Wang, Cao, Tang, Wuniqiemu, Teng, Zhu, Wei and Dong. This is an open-access article distributed under the terms of the Creative Commons Attribution License (CC BY). The use, distribution or reproduction in other forums is permitted, provided the original author(s) and the copyright owner(s) are credited and that the original publication in this journal is cited, in accordance with accepted academic practice. No use, distribution or reproduction is permitted which does not comply with these terms. 